\title{
Magnitude and Factors Affecting Parental Stress and Effective Stress Management Strategies Among Family Members During COVID-19
}

\author{
Usha Rani Kandula \\ Addisu Dabi Wake \\ Department of Nursing, College of \\ Health Sciences, Arsi University, Asella, \\ Oromia Regional State, Ethiopia
}

\begin{abstract}
The pandemic outbreak, coronavirus disease 2019 (COVID-19) threatens proper family patterns by disrupting normal day-to-day activities, such as working patterns of parents by working from home, online children's education rather than regular school, a dilemma in children's education and health constrained movement by complete or partial home lockdowns to prevent disease spread, anxiety of disease spread, failure of home quarantine due to insufficient home situation and reduced personal protective equipment usage due to the extra financial burden, reduced social communications, increased health burdens than usual along with enormous toil with dependent family members, demands of financial burdens by multiple factors, reduced monthly income during pandemics, lack of employment or job insecurity, deviations in mental health among family members such as fear, anxiety, insomnia, severe depression, hetero aggression, child verbal or physical abuses, increased alcohol consumption and substance abuse or drug addictions, aggressive behavior, self-blaming or injury, unintentional suicidal tendencies, mood disorders, posttraumatic stress disorder and failure to meet health demands due to a lack of health facilities. Many contributing factors are affecting the normal family status than usual circumstances such as reduced hospital equipment or resources which is due to pandemic effects, social isolation due to outside quarantine measures, insecurity of the future and ongoing pandemic situation, fear of vaccination side effects, and so on. Therefore, we hope to raise awareness about the magnitude of parental stress levels and contributing factors. Moreover, these findings would encourage effective stress management to overcome the factors that contribute to parenteral stress and to improve coping skills to face the coming darkness with ease. It supports the preservation of physical, mental, and social well-being in families as well as minimizing or overcoming stressors, maintaining strong familial ties, and improving life expectancy globally during this pandemic.
\end{abstract}

Keywords: COVID-19, factors, magnitude, novel, parental stress, stress management

\section{Introduction}

The COVID-19 epidemic has a devastating influence on people all across the world. It has been regarded as a global public health crisis that poses a threat to all elements of human life. ${ }^{1-4}$ The first cases of a novel coronavirus with pneumonia symptoms associated with severe acute respiratory syndrome coronavirus-2, were reported in Wuhan, China. ${ }^{4}$ The virus causes severe respiratory disease in humans when it spreads from an animal source to humans. ${ }^{5-8}$ People are infected with the deadly virus on a vast scale throughout the world. Many have perished as a result of
Correspondence: Usha Rani Kandula Department of Nursing, College of Health Sciences, Arsi University, P.O Box: 193/04, Asella, Oromia Regional State, Ethiopia

Emailneha20I0dec@gmail.com 
their incapacity to fight it. As a result, the world health organization (WHO) declares a global pandemic on March 12th, 2020. ${ }^{6-11}$

As of November 23, 2021, WHO had received 257,469,528 confirmed cases of COVID-19 with 5,158,211 fatalities. According to the most recent WHO figures, a total of 7,408,870,760 vaccine doses have been provided as of November 21, 2021. ${ }^{12}$ Real-time reverse transcription-polymerase chain reaction assays, examination of upper and lower respiratory tract secretions, endotracheal secretions, and additional body fluid examinations such as blood, urine, and feces, and possible radio diagnostic examinations for the confirmation of viral load are used to diagnose virus-affected patients. All patients in life-threatening situations are admitted to intensive care units for immediate illness management and breathing support to ensure the patient's survival. ${ }^{5}$

The viral spread is only controlled by isolating virusinfected patients, maintaining strict quarantine, and providing effective vaccination against the COVID-19 or Severe acute respiratory syndrome coronavirus- 2 virus to stabilize morbidity and mortality on the occurrence of virus-related deaths. Many countries have successfully created numerous types of immunizations and implemented them, and they are still working hard to manage the COVID-19 pandemic. $^{13}$

The WHO defines health as "a state of complete physical, mental, and social well-being and not merely the absence of disease or infirmity". ${ }^{14}$ As per the WHO health definition or statement, people are failed in the maintenance of normal life in all aspects during the COVID-19 pandemic era. A wide range of factors are affecting families around the globe due to the increasing burden of COVID-19. Parents are the primary caregivers of their children and fulfill the day-to-day needs of them concurrently. $^{15}$

The global pandemic constraints are bringing big challenges among parents and families by altering the regular activities of work or employment from the home, diminished economic status of the family, especially on maintenance of health, limited fulfilment of basic needs, closing of daycare centers especially who depend on them and burden of unemployment. ${ }^{3,16}$ Besides, it has a burden in security jobs along with increased dependent family members, deviation to offline schooling modes through online platforms like whatsApp, Google meet or zoom web applications with minimized internet facility in remote areas, failure to manage or assist the children education from home, parent work load increased with children new educational patterns, diminished health status of the family members with sudden pandemic attacks, and house arrest due to complete or partial lockdowns. ${ }^{6}$ Moreover, fear of pandemic spread among the family members or community, failure of home quarantine due to minimized home environment, minimal or improper usage of personal protective equipment's financial burdens, restricted personal social communication, diminished physical activity of the children, unusual verbal and physical abuse of the children by their parents, severe hetero aggressions among the families, and loneliness. ${ }^{3}$ Furthermore, isolation due to virus spread or outside quarantine practices, unfamiliar travel restrictions, minimal recreational activities, severe frustration on ongoing or uncertainty situation of pandemic conditions, failure to meet health demands of the family members by minimizing health resources during pandemic attack, failure to utilize health insurance services, unexpected deviations on mental health of family members by increased fear, anxiety, insomnia, severe stress, depression, ${ }^{4}$ self-blaming or injury, aggressive behavior, unintentional suicidal thoughts or tendencies, ${ }^{3,6,16}$ increased alcohol and substance abuse or drug addictions, ${ }^{3,17}$ sudden death of loved one in the family, mood disorders, ${ }^{18}$ and posttraumatic stress disorder/posttraumatic stress syndrome. ${ }^{4,6}$ These all contributing factors are creating a severe pandemic burden among

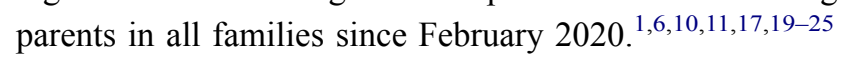

\section{Magnitude of Parent Stress}

The author recorded the magnitude of the parent's stress in the form of a review of the literature for an easy understanding of each study. Each review is explained on title of the study. The different scales were used to assess parental stress during the studies.

The study highlighted immediate psychological responses and associated factors during the beginning phases of COVID-19 among 1210 respondents. The psychological impact of the study was assessed by the event scale-revised (IES-R), and mental health status was assessed by the depression, anxiety, and stress scale 21 (DASS-21). According to this study findings, 53.8\% rated the psychological impact of the outbreak as moderate or severe. About $16.5 \%$ had moderate to severe depressive symptoms, $28.8 \%$ had moderate to severe anxiety symptoms, and $8.1 \%$ had moderate to severe stress levels. ${ }^{1}$

The study identified substantial changes in parents with an increased level of anxiety and depressive mood. 
A minimized interpersonal communicative relation may show a burden on child caring and child neglect among family members as well as other dependents of the family members. ${ }^{26}$ According to a report of a new study, the number of suicides in Canada connected with unemployment is expected to increase from 418 to $2114 .^{6}$ In addition to this, a systematic review and meta-analysis of a mental health impact of COVID-19 depression outcomes across countries was found to be $21.39 \%$ of study participants which had clinically significant depressive symptoms. ${ }^{23}$

A recent study on perceived stress as a mediator for longitudinal impacts of the COVID-19 lockdown on the well-being of parents and children found a significant change in parental negative feelings and over-reactivity during a COVID-19 pandemic with a total study sample of 106 parents. Parental stress was assessed using the perceived stress scale, which ranges from 0 to 4 . Parental stress was found to be between 0 and 2.4 among parents. ${ }^{26}$

A new study looked at the impact of autism spectrum disorder (ASD) on parents' stress and emotional wellbeing during the COVID-19 pandemic using quantitative observations of 150 parents of children with ASD who had a substantial influence on parental stress and emotional well-being. In this study, $94 \%$ of parents were found to be stressed, and $78.7 \%$ of parents' mental well-being was impaired during COVID-19. ${ }^{27}$ Stress and parenting were identified as risk factors for child maltreatment during the global COVID-19 pandemic. The study's findings revealed a greater level of perceived parental stress of $6.59 \% .{ }^{25}$

During a COVID19 pandemic, a research study found links between parental stress, parent mental health, and prevalence of unfavorable childhood experiences in families. According to the findings of a representative poll of 1024 parents, more than half of the parents feel anxious as a result of social isolation (56.1\%), school closures $(52.1 \%)$, and a shortage of childcare services $(52.1 \%){ }^{15}$

Research evidence identified on the mediating effects of parental stress on harsh parenting and parent child relationship during COVID-19 pandemic parental stress among 258 members. The recognized mean parental stress was 2.40 with a standard deviation of 0.46 and a range was $2.50 .{ }^{28}$ A study conducted on Italian parents among 463 members on stress, resilience, and well-being among Italian children and their parents during the COVID-19 pandemic. The study declared with the parents who are living with physical or psychological problems of children was under high parent stress scale scores as mean $=42.3$, standard error $(\mathrm{SE})=1.74$ when compared to parents who are living with healthy children with parent stress scale score of mean=36.6, $\mathrm{SE}=1.14 .^{29}$ A study conducted in Italy on the effect of COVID-19 lockdown on parents among 1126 parents of children who are suffering from mental or physical disorders are perceived severe stress and parental burden $\mathrm{t}^{1124}-2.70, \mathrm{p}-0.001 .^{30}$

The research conducted in Canada on the examination of family mental health during COVID-19 reported that during the first lockdown period in Canada, $44.3 \%$ of parents of children below the age of 18 years who are living at home are identified with severe mental illness during the pandemic situation. ${ }^{31}$ The study regarding parental stress before COVID-19 revealed a mean score of 13.09 with SD of 4.61 and when compared to post COVID-19 mean score which was 13.49 with SD of $4.98 .^{32}$ A study was conducted on parents' stress and children's psychological problems in families facing COVID-19 outbreak in Italy. This study was emphasized on parenting stress index mean was 22.01 with SD was 7.65 and also study analyzed regarding parental DASS mean was 29.57 with SD was $10.28 .^{33}$

The study was conducted on the parent stress level in facing children studied from home in the early of COVID19 pandemic in Indonesia among 223 members. The findings of this study explored the stress levels of parents. From this, $75.34 \%$ of parents were experienced moderate stress of which, $71.88 \%$ of respondents were women who experienced high stress levels. ${ }^{19} \mathrm{~A}$ research study done on the vulnerability and resilience in children during the COVID-19 pandemic among 29,202 parents of Hong Kong revealed that the identified parental stress mean was 46.74 with SD being $10.35 .{ }^{34}$ The study identified that the detection rates of depression and anxiety in parents were $6.1 \%$ and $4.0 \%$, respectively. The perceived stress levels of parents are higher in families with internal conflicts when compared with normal healthy families. ${ }^{35}$

A study was reported among Americans, Asians, and Europeans using the chain mediation model to COVID-19 symptoms and mental health outcomes with a crosssectional research design among 4612 participants. The study instruments are the IES-R questionnaire and DASS-21. According to the findings, Poland and the Philippines had the highest levels of anxiety, depression, and stress, while Vietnam had the lowest mean scores in these areas. The overall scores with mean and SD were perceived psychological impact of COVID-19: mean \pm SD 
(2.24 \pm 1.27$)$; DASS-21 stress: mean \pm SD $(1.75 \pm 1.03)$; DASS-2 1 anxiety: mean \pm SD $(1.90 \pm 1.36)$; DASS-21 depression: mean $\pm \mathrm{SD}(1.78 \pm 1.21)$; physical symptoms resembling COVID-19 infection: mean \pm SD (1.06 \pm 1.43); the need for health information: mean \pm SD (7.58 $\pm 3.42){ }^{10}$

The impact of the COVID-19 pandemic on Asians' physical and mental health was investigated in a study among the 4479 respondents. A study was conducted on seven Asian middle-income countries. To assess mental health, the IES-R and DASS-21 were used. The respondents filled out a questionnaire with demographic information, physical symptoms, and healthcare utilization, contact history, knowledge, and concerns, and precautionary measures, as well as completing the IES-R and DASS21 to rate their mental health. Protective and risk factors linked with mental health indicators were identified using descriptive statistics, one-way analysis of variance and linear regression. Between 7 middle income countries, there were significant differences in IES-R and DASS-21 scores $(\mathrm{p}<0.05)$. Thailand received the highest IES-R and DASS-21 stress, anxiety, and depression levels while Vietnam received the lowest. ${ }^{22}$

\section{Factors Affecting Parental Stress}

Many contributing factors are directly or indirectly causing increased parental stress during COVID-19 pandemic. There are different factors which were identified from the literature by addressing parental stress.

\section{Parental Negative Feelings}

The majority of the parents are identified with exhibiting negative feelings or psychological disturbances like depression, anxiety, frustration, confusion, over or hyperreactivity, interpersonal sensitivity, irritability, and disturbed sleep. ${ }^{4,15,25,26}$

\section{Hetero Aggressions or Domestic Violence}

A stressful environment of the family, increased family or domestic violence in the family during the pandemic crisis for various reasons such as failure to meet the demands of the dependent family members, unable to manage homerelated activities with added work during the pandemic as homeschooling with work from home, complete house arrest of the children, unable to meet the primary needs of the children in time, misunderstandings among parents, siblings complications, home quarantine or isolation of infected people with limited home spacing's are identified factors for the domestic violence during the pandemic era. $^{15,26,34,36}$

\section{The Burden of Physically Challenged People}

Several studies reported that parents are overburdened while caring for physically challenged people, especially autism spectrum disorder (ASD), and experience deficient emotional well-being with fluctuation of regular activities of the family, frustrations with child behaviors, unable to meet the demands of the children, intolerance with aggressive behavior of the children, more burden with single parenting child, minimized social supports during the pandemic. ${ }^{27,30,35}$

\section{Child Care Neglect or Maltreatment}

During a pandemic, parents are exposed to internal and external stressing factors. In some families, it showed a contributing impact on child maltreatment and child neglect or abuse and harsh parenting. ${ }^{25,36}$ Child maltreatment may be due to parental low education, single parenthood, economical problems causing poor parenting behavior towards child care and child maltreatment is identified more in families who are directly fighting with the deadly virus. ${ }^{32,37}$

\section{Increased Economic Burden}

For human survival, financial support will play a crucial role in the day-to-day life of every family. Increased demand of economic burden in families was due to COVID-19 health risk, failure to meet free health care services during a pandemic, unable to obtain health insurance services from the health insurance agencies due to demand of the health burden to the population at the time, economic crisis to receive private organizational services due to high-cost medical services and with limited family economic supports, and failure to grab the job opportunity in this critical times. ${ }^{2,20,25,30,32}$

\section{Pandemic Related Stress}

Pandemic related stress were due to sudden loss of family members, friends, relatives and colleagues, neighbors or family members identified with positive of the COVID-19 virus, stress with social distancing and limited social supports, home or hospital quarantine with social isolation, minimized 
social relations or communication, exposure to posttraumatic stress disorder during the critical times. ${ }^{4,15,33,38,39}$

\section{Loss of Employment}

Many families lose their jobs during the pandemic era or receive of half-pay scale from the employer due to decreased financial sources from the institutions of the working agencies, which are additional added financial stress among the families. Moreover, job opportunities were minimized for the newly graduated students in the family. This led to a failure to meet the demands of the families as necessary needs of the day-to-day life of people in a society. ${ }^{4,15,30,33}$

\section{Altered Children Education}

Sudden changes happened such as school closings and changes in the educational pattern of the children such as online mode delivery of the education system. Children are unable to cope with the changes in the educational pattern and sometimes unable to grasp the subject learning systems. Changes and difficulties in the pattern of public examination systems among children and adolescents, fear of viral contamination through school reopening, and final examination of children were the leading contributing factors for parental stress. ${ }^{1,2,4,30,33,40}$

\section{Minimized Education of Parents}

With less educational level of the parents are unable to assist their children during the online class timing. Some parents are unable to understand the medium of the language which is delivered by the school teachers. This inability to complete school homework is found to be the additional burden of home-related work. ${ }^{19,20,30}$

\section{Cellular or Smartphone Addiction of the Children}

Currently, all children are using smart mobile phones because of the online lecture deliveries. Sometimes children are misleaded towards online gaming, listening to songs from YouTube or watching videos, and engaging in whatsApp chatting with friends. Prolonging these activities may harm education concentration, which is a factor for parental stress. ${ }^{34,36}$

\section{Poor Parental Control of the Children}

Some parents are burdened by increased home-related responsibilities and are unable to regulate their children's activities, which can lead to misinformed behavior and full distraction from social connections with friends and other leisure activities. When children are suddenly exposed to the pandemic virus, some families are unable to care for them. ${ }^{19,29,41}$

\section{Diminished Psychological Well-Being}

Suddenly, people have come across phobia with pandemic exposure. Social deviations among the population, minimized communication with familiar people, reduced recreational activities, pandemic isolation, loneliness, frustration with regular usage of face masks, or unable to manage the face mask with respiratory suffocations, moisture covering with a face mask if a person has eyeglasses, finance burden with continuous usage of personal protective equipment by low-income family members, and decreased quality of life of the people especially in vulnerable groups during pandemics are may be distractive factors for the low psychological well-being or mental health disturbances among the people. , $^{2,30,31,35,42,43}$

\section{Closing of Schools and Child Care Centers}

For complete lockdown reasons, complete closure of children's school and the primary child care centers are under complete resting position. By this, some parents are unable to manage their children at home with behavioral changes among the children, inability to manage conflicts between siblings, and difficulty to continue their official work from the home environments. ${ }^{2,4,30,32}$

\section{Health Crisis}

The pandemic situation strongly affects the mental health of the people. Once in a family, anybody is affected by the virus and the health condition impacts the whole family. If the person is hospitalized in a family, it leads to an altered family process and all regular activities of the family are disturbed. ${ }^{30}$ Some parents are addicted to substance abuse, increasing thoughts towards suicidal tendencies, and selfharming or self-abusing themselves because of the increased psychological burden on them. ${ }^{4,31}$

\section{Failure to Meet Coping Strategies}

During pandemic lockdowns, all family members are sticking to home environments with limited home space, limited social communication, unable to meet relatives and friends, and failure to visit recreational areas such as 
shopping malls, visiting parks, movie theatres, and long driving with belongings. ${ }^{36}$

\section{Parental Separation from Children}

Parents are separated from their children if the parents are infected with the virus. For prevention of cross-infection from parent to child, parents are under the control of selfisolation or in the quarantine process. ${ }^{33,36}$

\section{Increased Burden of Single Parenthood}

Parents who are separated or divorced or living alone with children due to any circumstances are identified with more severe stress due to increased burden with domestic work, offline schooling, job-related work from home, and with minimized social supports, problems with siblings, and unable to compromise them, minimized financial burden, dependence of increased number of children's, and handling of any situation alone by the single parents are undergoing severe stress during the pandemic conditions. ${ }^{4,34}$

\section{Emotional Disturbances}

Emotional instability due to loneliness, inability to meet familiar coping mechanism skills of family members, and increased mental and physical health causes during the pandemics. ${ }^{4,19,36}$

\section{Deviation from Normal Life}

Some parents depend on alcohol substances or drug addiction, especially by men. The increased suicidal thoughts or self-harm and self-abusing among people were due to the inability to control the terrific situations of the pandemic crisis. $^{4,31}$

\section{Failure to Meet Health Emergencies}

Vulnerable groups like children, aging people, and especially pregnant women were unable to receive emergency care immediately due to a lack of health care personnel and services, which may lead to decreased quality of people and may progress to high-risk cases during the pandemic era. ${ }^{43}$

\section{Stress Management Strategies}

COVID-19 still influences some countries by different variants and dramatically influences health, economic, and social distractions. Due to the influence of this pandemic, people are frustrated around the world, especially highly influencing the increased burden on the parents by various contributing factors. Regular follow or maintenance of stress management strategies are keeping away of parents and other family members stress-free during pandemic vulnerability and sustained towards the healthy well-being.

\section{Family Adherence to Positive Coping Skills}

All family members should adopt to the inculcation of positive coping strategies and maintenance of supportive family environments, especially during the pandemic critical situation to prevent extreme stress among the family members. ${ }^{25,36}$

\section{Maintenance of Parent-Child Bonding}

Parents should understand the current situation of the pandemic and should maintain positive parent-child bonds towards their beloved children and try to fulfill the primary needs of their children. ${ }^{35,36}$

\section{Expanding Communications}

Following effective communication with peer groups while maintaining social distancing, people may relax and be free from pandemic stress and by sharing proper health information on COVID-19 preventive protocols. ${ }^{44}$

\section{Adequate Sleeping}

Adequate sleeping practices induce physical and mental relaxation in this present situation. Maintenance of adequate sleeping practices among the family members will help to forget the fear of COVID-19 pandemic situation..$^{19,39}$

\section{Follow-Up Regular Exercises}

The performance of regular exercises will improve the physical and mental strength of the people. Regular performance of physical activity or exercise by family members during COVID-19 will provide positive energy and healthy lifestyle during the pandemic time. ${ }^{45}$

\section{Increasing Self-Care Practices}

Self-care practices are essential to maintain the positive health of each individual in a family. Following and guiding self-care practices among family members during the pandemic time will help to maintain the body fitness of the people and away from the illness. ${ }^{39}$

\section{Increasing Quality of Life}

Day by day, increasing the quality of life by focusing positive thoughts, self-reading, listening to music, 
spending time with family members, storytelling to the children, spending time with spirituality, and maintenance of physical fitness will progress towards the maintenance of the quality of people life during the pandemic era. ${ }^{19,39}$

\section{Minimizing Conflicts}

By minimizing day-to-day life stress, anxiety, depression, and other negative feelings, people can maintain positive mental well-being. Minimizing interpersonal conflicts and trying to maintain healthy relations among people will improve the positive strength among people. ${ }^{25}$

\section{Obtaining Possible Social Support}

During a partial or complete lockdown due to a pandemic, there is a gap between the maintenance of social communication. People can maintain social support from family members, friends, and neighbors by extending telecommunication, audio, or video calls, can share their feelings and droughts, and adjust with social supports as much as available during this time till the recovery period. ${ }^{36}$

\section{Equal Work Delegation}

Adjusting the timing of home activities, managing domestic work among family members and professional work burden, and maintaining a good professional connectedness with family members, friends, and co-workers may minimize pandemic-related stress. ${ }^{39}$

\section{Increasing of Self-Awareness}

Increasing self-awareness towards the pandemic situation, knowing the present family members' health conditions, and may increase the effective communication with family as well as connect surrounding people to the know present pandemic awareness and follow preventive measures towards the deadly virus. ${ }^{39}$

\section{Obtaining Counseling Services}

In a family, if any special needs exist, they should undergo counseling services to increase the optimal health and minimize confusion and choose a better possible option from the counseling services and increase the wellness of the family. ${ }^{19}$

\section{Follow-Up Health Care Services}

Pandemics brought health crisis among the world. Following health care services, if the need identifies or to support a dependent families, peoples will reduce the health burden during critical times. ${ }^{46}$

\section{Fulfilling the Basic Needs of the Family}

Primarily understand the dependent family member's conditions and their needs. Fulfilling the basic needs of the family members will improve the family's well-being during pandemics. ${ }^{31}$

\section{Performing Stress Reducing Activities}

Following possible stress-reducing activities by all family members such as storytelling to the children's, encouraging play activities by the children's, engaging with subject matter, performance of dance activities, maintaining good time with the family, following yoga and meditation, and obtaining spiritual assistance among the family members, if possible watching television and knowing the situation of the pandemics by updating the current health information, and following laughing therapies by watching comedies could be the stress reducing activities. ${ }^{36,47}$

\section{Managing Financial Status of the Family}

Effective managing of financial family needs by reducing unnecessary spending on shopping, identifying the available budget in a month and planning monthly spending, and identifying the financial burdens in the family and trying to adjust the family economic status are strategies to manage the financial status of the family. ${ }^{31}$

\section{Utilizing Health Care Facilities}

Health problems can be solved only by approaching the health care sector or undergoing proper health care services. Utilizing free health-related facilities if the need persists among families may assist the physical and financial burden during critical times. ${ }^{25}$

\section{Adopting a Positive Work Environment}

This includes maintenance of a positive work environment at the working area as well as home environments, schedule work and minimize workload and focus on the comprehensive life among everyone, adapting resting hours by minimizing severe workload and stress, and seeking continuous health care support if the emergency persists. ${ }^{48}$

\section{Increasing Self-Confidence}

Good progress will occur by increasing self-confidence in all activities, day by day improving adaptive coping and cognitive skills, adjusting for problem-solving behaviors, following strategies for improving family members' health, and improving job satisfaction. ${ }^{39}$ 


\section{Assisting with the Peer Group}

Sometimes peer support will help to reduce mental stress during a pandemic. Assist with peer support to remove the extreme barriers in all aspects of physical, mental, and social well-being of family members by sharing or exchanging ideas during pandemic phobia time. ${ }^{25}$

\section{Boosting Immunity Towards COVID-I 9}

Utilizing the vaccination facilities to boost up the immunity and by following benefiting healthy tips like good nutrition support, vitamin-c supplements, drinking of hot water and minimizing respiratory infections, following isolation practices, support for medical emergencies, following individual relaxation techniques to minimize perceived stress, attending COVID-19 awareness programs, increasing of selfefficacy skills towards the prevention of the COVID-19, and ready for the self towards the upcoming problems. ${ }^{31,32}$

\section{Increasing Emotional Stability During}

\section{a Pandemic}

Increasing emotional stability towards uncertain events or upcoming challenges, increasing psychological well-being among sick people, and reducing anxiety levels with spiritual encouragement. ${ }^{45}$

\section{Assisting of Dependent Family Members}

Family is very essential in the community. Some family members are dependent on a family for various reasons such as children with less age or adults with increased age and some dependent on health deviation situations. Physical aiding of dependent family members in fulfilling their needs, improving decision-making skills in any situation will progress a positive and healthy family in any difficult situation. ${ }^{39}$

\section{Adopting Independent Decision-Making Skills}

Parents should be independent in any situation for proper handling of any critical situation such as physical, financial, and social problems. They should be ready enough to take a competent decision towards their family members during the tough times to save their family members during emergency lifesaving conditions. ${ }^{30}$

\section{Following COVID-19 Protocols}

Without our negligence following the COVID-19 protocol, minimizing traveling activities to reduce the spread of infection and help to prevent health threats of pandemics, control or minimizing internal and external stressing factors, ready to engage in routine activities of day to day life for the better enhancement of life, following social distancing, avoidance of the mass gatherings, and following preventive measures towards the pandemic prevention. ${ }^{19,45}$

\section{Adopting Healthy Behaviors}

Avoidance of harmful activities by people to support family members as alcohol dependency, cigarette smoking, and any ill-health behaviors. ${ }^{51}$

\section{Ability to Manage the Child Behaviors}

Childhood is a precious time for growing children. Assisting children's routine activities as well as educational needs and minimizing the burden of digital technologies on children. During a pandemic, children are encouraged towards play and art activities along with education to prevent the burden and increase the interest of children towards the positive enhancement towards the surroundings of the children's. ${ }^{19,36}$

\section{Away from the Illness Behaviors}

Away from health illness behaviors such as alcohol consumption or drug addiction, self-harm, and self-abusing and prevent neglecting behavior towards family and friends and prevent health-related complications towards family members. Measures to take for the maintenance of physical health and prevention of the disease and improve the good health of the family members. ${ }^{51}$

\section{Understanding Loss and Grief}

Understand and cope and adjust to the loss of being loving or family members adopting to the optimist towards the upcoming life. ${ }^{52}$

\section{Focusing on the Future}

Increasing the concentration towards present activities and not criticizing the present situation or to the people, increasing coping with upcoming challenges in the future, acquiring competent skills for the handling of current situations, and positive hope for the future, and focusing on overcoming life-threatening barriers. ${ }^{51}$

\section{Following the Plan of Action}

Create a plan of action for upcoming uncertain situations and effectively implement the plan of activities as well as 
maintain social distance from the onset of upcoming illness or health threats. The attempt to connect people through social networking and managing effective communication for the preservation of healthy human relations, raising awareness among family members about the spread of a deadly virus, and engaging in problemsolving strategies would best as an action plan. ${ }^{51,53}$

\section{Exposure to Frequent Recreational Activities}

Listening to music for recreational activities, focusing on essential work, caring for being loving's, and engaging in outdoor activities as gardening may be away from pandemic stress. $^{45,54}$

\section{Focusing or Follow-Up Psychological Interventions}

During a pandemic, many people undergo severe psychological burdens as fear, anxiety, depression, and suicidal thoughts. These groups are advised to undergo psychological interventions as mind-body relaxation techniques, psycho-education, psychological counseling, or the need to provide psychotherapies like cognitive behavior therapy and mindfulness-based cognitive therapy to overcome the mental health changes which are occurring during the pandemic. The cognitive behavior therapy services may be obtained through online platforms during difficult times also. These techniques may help people to combat the mental health conditions of people during pandemics. ${ }^{11,55,56}$

\section{Conclusion}

Since the beginning of the year 2020 and until now, worldwide people are exposed to the unfamiliarity situation as not to wish to expose in the forthcoming years. The impact of COVID-19 drastically destroyed parental well-being through creating various stressing factors. The pandemic crisis explored the negative impact on the parents and their family members by deviating all normal day activities of family, community, society, etc. Till now, many countries are unable to fight the pandemic critically and parents are undergoing many intolerable situations, especially by low and middle-income family people. In these critical times, the governments of each country need to understand the parental stress and try to minimize the parental stress by assisting or implementing different parental needy activities. This could include providing financial assistance, extending facilities towards availability of free corporate health services on an emergency basis, and increasing pandemic awareness and preventing protocols among people. Parents and family members essentially know the stress management techniques during the pandemic, to be away from highly stressful conditions and may undergo psychological interventions, and to be competent to tackle any tough time effectively by minimizing stressors and increasing hope towards the upcoming future and trying to inculcate to build healthy families, communities, etc.

\section{Author Contributions}

Both authors have made a significant contribution to conception and design, acquisition of data, or analysis and interpretation of data; took part in drafting, revising or critically reviewing the article; gave final approval of the version to be published; and agrees to be accountable for all aspects of the work.

\section{Funding}

There was no budget allocated or financial support for this study.

\section{Disclosure}

The authors declared that there is no conflict of interest related to this work.

\section{References}

1. Wang C, Pan R, Wan X, et al. Immediate psychological responses and associated factors during the initial stage of the 2019 Coronavirus Disease (COVID-19) epidemic among the general population in China. Int $J$ Environ Res Public Health. 2020;17(5):E1729. doi:10.3390/ijerph17051729

2. Tran BX, Nguyen HT, Le HT, et al. Impact of COVID-19 on economic well-being and quality of life of the Vietnamese during the national social distancing. Front Psychol. 2020;11:565153. doi:10.3389/ fpsyg.2020.565153

3. Costanza A, Marco SD, Burroni M, et al. Meaning in life and demoralization: a mental-health reading perspective of suicidality in the time of COVID-19. Acta Biomed Atenei Parm. 2020;91(4):e2020163.

4. Ren Z, Xin Y, Ge J, et al. Psychological impact of COVID-19 on college students after school reopening: a cross-sectional study based on machine learning. Front Psychol. 2021;12:641806. doi:10.3389/ fpsyg.2021.641806

5. Bouadma L, Lescure F-X, Lucet J-C, Yazdanpanah Y, Timsit J-F. Severe SARS-CoV-2 infections: practical considerations and management strategy for intensivists. Intensive Care Med. 2020;46 (4):579-582. doi:10.1007/s00134-020-05967-x

6. Xiong J, Lipsitz O, Nasri F, et al. Impact of COVID-19 pandemic on mental health in the general population: a systematic review. $J$ Affect Disord. 2020;277:55-64. doi:10.1016/j.jad.2020.08.001

7. Anand KB, Karade S, Sen S, Gupta RM. SARS-CoV-2: Camazotz's curse. Med J Armed Forces India. 2020;76(2):136-141. doi:10.1016/j. mjafi.2020.04.008 
8. Orgilés M, Morales A, Delvecchio E, Mazzeschi C, Espada JP. Immediate psychological effects of the COVID-19 quarantine in youth from Italy and Spain. Front Psychol. 2020;11:2986. doi:10.3389/fpsyg.2020.579038

9. Ciotti M, Ciccozzi M, Terrinoni A, Jiang W-C, Wang CB, Bernardini S. The COVID-19 pandemic. Crit Rev Clin Lab Sci. 2020;57(6):365-388. doi:10.1080/10408363.2020.1783198

10. Wang C, Chudzicka-Czupała A, Tee ML, et al. A chain mediation model on COVID-19 symptoms and mental health outcomes in Americans, Asians, and Europeans. Sci Rep. 2021;11(1):6481. doi:10.1038/s41598-021-85943-7

11. Ho CS, Chee CY, Ho RC. Mental health strategies to combat the psychological impact of coronavirus disease 2019 (COVID-19) beyond paranoia and panic. Ann Acad Med Singapore. 2020;49 (3):155-160. doi:10.47102/annals-acadmedsg.202043

12. World Health Organization. WHO Coronavirus (COVID-19) dashboard [Internet]. [cited November 24, 2021]. Available from: https:// covid19.who.int. Accessed December 31, 2021.

13. Amanat F, Krammer F. SARS-CoV-2 vaccines: status report. Immunity. 2020;52(4):583-589. doi:10.1016/j.immuni.2020.03.007

14. Health knowledge. Section 3: concepts of health and wellbeing [Internet]. Health Knowledge; 2010 [cited July 20, 2021]. Available from: https://www.healthknowledge.org.uk/public-health-textbook /medical-sociology-policy-economics/4a-concepts-health-illness/sec tion2/activity3. Accessed December 31, 2021.

15. Calvano C, Engelke L, Di Bella J, Kindermann J, Renneberg B, Winter SM. Families in the COVID-19 pandemic: parental stress, parent mental health and the occurrence of adverse childhood experiences-results of a representative survey in Germany. Eur Child Adolesc Psychiatry. 2021. doi:10.1007/s00787-021-01739-0

16. McIntyre RS, Lee Y. Preventing suicide in the context of the COVID-19 pandemic. World Psychiatry. 2020;19(2):250-251. doi: 10.1002/wps. 20767

17. Costanza A, Rothen S, Achab S, et al. Impulsivity and impulsivity-related endophenotypes in suicidal patients with substance use disorders: an exploratory study. Int $J$ Ment Health Addict. 2020. doi:10.1007/s11469-020-00259-3

18. Ceban F, Nogo D, Carvalho IP, et al. Association between mood disorders and risk of COVID-19 infection, hospitalization, and death: a systematic review and meta-analysis. JAMA Psychiatry. 2021;78 (10):1079-1091. doi:10.1001/jamapsychiatry.2021.1818

19. Susilowati E, Azzasyofia M. The parents stress level in facing children study from home in the early of COVID-19 pandemic in Indonesia. Int J Sci Soc. 2020;2(3):1-12. doi:10.54783/ijsoc.v2i3.117

20. Cluver L, Lachman JM, Sherr L, et al. Parenting in a time of COVID-19. Lancet Lond Engl. 2020;395(10231):e64. doi:10.1016/ S0140-6736(20)30736-4

21. Clemens V, Deschamps P, Fegert JM, et al. Potential effects of "social" distancing measures and school lockdown on child and adolescent mental health. Eur Child Adolesc Psychiatry. 2020;29 (6):739-742. doi:10.1007/s00787-020-01549-w

22. Wang C, Tee M, Roy AE, et al. The impact of COVID-19 pandemic on the physical and mental health of Asians: a study of seven middle-income countries in Asia. PLoS One. 2021;16(2):e0246824. doi:10.1371/journal.pone.0246824

23. Lee Y, Lui LMW, Chen-Li D, et al. Government response moderates the mental health impact of COVID-19: a systematic review and meta-analysis of depression outcomes across countries. J Affect Disord. 2021;290:364-377. doi:10.1016/j.jad.2021.04.050

24. Mazza MG, De Lorenzo R, Conte C, et al. Anxiety and depression in COVID-19 survivors: role of inflammatory and clinical predictors. Brain Behav Immun. 2020;89:594-600. doi:10.1016/j.bbi.2020. 07.037

25. Brown SM, Doom JR, Lechuga-Peña S, Watamura SE, Koppels T. Stress and parenting during the global COVID-19 pandemic. Child Abuse Negl. 2020;110:104699. doi:10.1016/j.chiabu.2020.104699
26. Achterberg M, Dobbelaar S, Boer OD, Crone EA. Perceived stress as a mediator for longitudinal effects of the COVID-19 lockdown on the wellbeing of parents and children. Sci Rep. 2021;11(1). doi:10.1038/ s41598-021-81720-8

27. Alhuzimi T. Stress and emotional wellbeing of parents due to change in routine for children with Autism Spectrum Disorder (ASD) at home during COVID-19 pandemic in Saudi Arabia. Res Dev Disabil. 2020;108:103822. doi:10.1016/j.ridd.2020.103822

28. Chung G, Lanier P, Wong PYJ. Mediating effects of parental stress on harsh parenting and parent-child relationship during coronavirus (COVID-19) pandemic in Singapore. J Fam Violence. 2020;2:1-12.

29. Cusinato M, Iannattone $\mathrm{S}$, Spoto $\mathrm{A}$, et al. Stress, resilience, and well-being in Italian children and their parents during the COVID-19 pandemic. Int J Environ Res Public Health. 2020;17 (22):8297. doi:10.3390/ijerph17228297

30. Fontanesi L, Marchetti D, Mazza C, Di Giandomenico S, Roma P, Verrocchio MC. The effect of the COVID-19 lockdown on parents: a call to adopt urgent measures. Psychol Trauma Theory Res Pract Policy. 2020;12(S1):S79-S81. doi:10.1037/tra0000672

31. Gadermann AC, Thomson KC, Richardson CG, et al. Examining the impacts of the COVID-19 pandemic on family mental health in Canada: findings from a national cross-sectional study. BMJ Open. 2021;11(1):e042871. doi:10.1136/BMJ.open-2020-042871

32. Miller JJ, Cooley ME, Mihalec-Adkins BP. Examining the Impact of COVID-19 on parental stress: a study of foster parents. Child Adolesc Soc Work J. 2020. doi:10.1007/s10560-020-00725-w

33. Spinelli M, Lionetti F, Pastore M, Fasolo M. Parents' stress and children's psychological problems in families facing the COVID-19 outbreak in Italy. Front Psychol. 2020;11. doi:10.3389/fpsyg. 2020.01713

34. Tso WW, Wong RS, Tung KT, et al. Vulnerability and resilience in children during the COVID-19 pandemic. Eur Child Adolesc Psychiatry. 2020;29:1-16. doi:10.1007/s00787-020-01470-2

35. Wu M, Xu W, Yao Y, et al. Mental health status of students' parents during COVID-19 pandemic and its influence factors. Gen Psychiatry. 2020;33(4):e100250. doi:10.1136/gpsych-2020-100250

36. Imran N, Zeshan M, Pervaiz Z. Mental health considerations for children \& adolescents in COVID-19 pandemic. Pak J Med Sci. 2020;36(COVID19-S4):S67-S72. doi:10.12669/pjms.36.COVID19S4.2759

37. Hutchison L, Feder M, Abar B, Winsler A. Relations between parenting stress, parenting style, and child executive functioning for children with ADHD or autism. J Child Fam Stud. 2016;25 (12):3644-3656. doi:10.1007/s10826-016-0518-2

38. Brooks SK, Webster RK, Smith LE, et al. The psychological impact of quarantine and how to reduce it: a rapid review of the evidence. Lancet. 2020;395(10227):912-920. doi:10.1016/S0140-6736(20)30460-8

39. Heath C, Sommerfield A, von Ungern-sternberg BS. Resilience strategies to manage psychological distress among healthcare workers during the COVID-19 pandemic: a narrative review. Anaesthesia. 2020;75(10):1364-1371. doi:10.1111/anae.15180

40. Pajarianto H, Kadir A, Galugu N, Sari P. Study from home in the middle of the COVID-19 pandemic: analysis of religiosity, teacher, and parents support against academic stress. 2020:1791-1807.

41. Waldfogel J, Craigie T-A, Brooks-Gunn J. Fragile families and child wellbeing. Future Child. 2010;20(2):87-112. doi:10.1353/foc.2010.0002

42. Wang C, Chudzicka-Czupała A, Grabowski D, et al. The association between physical and mental health and face mask use during the COVID-19 pandemic: a comparison of two countries with different views and practices. Front Psychiatry. 2020;11:569981. doi:10.3389/ fpsyt.2020.569981

43. Nguyen LH, Nguyen LD, Ninh LT, et al. COVID-19 and delayed antenatal care impaired pregnant women's quality of life and psychological well-being: what supports should be provided? Evidence from Vietnam. J Affect Disord . 2021;298(Pt A):119-125. doi:10.1016/j. jad.2021.10.102 
44. Dijkstra MTM, Homan AC. Engaging in rather than disengaging from stress: effective coping and perceived control. Front Psychol. 2016;7. doi:10.3389/fpsyg.2016.01415

45. Vogel EA, Zhang JS, Peng K, et al. Physical activity and stress management during COVID-19: a longitudinal survey study. Psychol Health. 2021:1-11. doi:10.1080/08870446.2020.1869740

46. Maunder RG, Leszcz M, Savage D, et al. Applying the lessons of SARS to pandemic influenza: an evidence-based approach to mitigating the stress experienced by healthcare workers. Can J Public Health. 2008;99(6):486-488. doi:10.1007/BF03403782

47. Vidas D, Larwood JL, Nelson NL, Dingle GA. Music listening as a strategy for managing COVID-19 stress in first-year university students. Front Psychol. 2021;12:647065. doi:10.3389/fpsyg.2021. 647065

48. Callahan K, Christman G, Battling Burnout: ML. Strategies for promoting physician wellness. Adv Pediatr. 2018;65(1):1-17. doi:10.1016/j.yapd.2018.03.001

49. Schreiber M, Cates DS, Formanski S, King M. Maximizing the resilience of healthcare workers in multi-hazard events: lessons from the 2014-2015 Ebola response in Africa. Mil Med. 2019;184 (Suppl 1):114-120. doi:10.1093/milmed/usy400

50. Mahmoud NN, Rothenberger D. From burnout to well-being: a focus on resilience. Clin Colon Rectal Surg. 2019;32(6):415-423. doi:10. 1055/s-0039-1692710
51. Lupe SE, Keefer L, Szigethy E. Gaining resilience and reducing stress in the age of COVID-19. Curr Opin Gastroenterol. 2020;36(4):295-303. doi:10.1097/MOG.00000000 00000646

52. Klapproth F, Federkeil L, Henschke F, et al. Teachers experiences of stress and their coping strategies during COVID - 19 induced distance teaching. J Pedagog Res. 2020;4(4):444-452. doi:10.33902/ JPR.2020062805

53. Mattioli AV, Sciomer S, Maffei S, Gallina S. Lifestyle and stress management in women during COVID-19 pandemic: impact on cardiovascular risk burden. Am J Lifestyle Med. 2021;15 (3):356-359. doi:10.1177/1559827620981014

54. Rama-Maceiras P, Jokinen J, Kranke P. Stress and burnout in anesthesia: a real-world problem? Curr Opin Anaesthesiol. 2015;28 (2):151-158. doi:10.1097/ACO.0000000000000169

55. Zhang MWB, Ho RCM. Moodle: the cost-effective solution for internet cognitive behavioral therapy (I-CBT) interventions. Technol Health Care. 2017;25(1):163-165.

56. Soh HL, Ho RC, Ho CS, Tam WW. Efficacy of digital cognitive behavioral therapy for insomnia: a meta-analysis of randomized controlled trials. Sleep Med. 2020;75:315-325. doi:10.1016/j.sleep.20 20.08.020
Psychology Research and Behavior Management

\section{Publish your work in this journal}

Psychology Research and Behavior Management is an international, peer-reviewed, open access journal focusing on the science of psychology and its application in behavior management to develop improved outcomes in the clinical, educational, sports and business arenas. Specific topics covered in the journal include: Neuroscience, memory and decision making; Behavior modification and management; Clinica

\section{Dovepress}

applications; Business and sports performance management; Social and developmental studies; Animal studies. The manuscript management system is completely online and includes a very quick and fair peer-review system, which is all easy to use. Visit http://www. dovepress.com/testimonials.php to read real quotes from published authors. 